\title{
STABILITY OF EQUIVARIANT SMOOTH MAPS
}

\author{
BY V. POÉNARU
}

Communicated May 6, 1975

This research announcement is a summary of a paper which will appear elsewhere [5], and which continues the program started in [4].

1. We consider a compact Lie group $G$ and smooth compact $G$-manifolds $X$ and $Y$. By $C_{G}^{\infty}(X, Y)$, Diff ${ }_{G}(X), \operatorname{Diff}_{G}(Y)$ we denote the $C^{\infty}$, Gequivariant mappings $X \rightarrow Y$, respectively, diffeos of $X$ or diffeos of $Y$.

There is a natural group action

$$
\operatorname{Diff}_{G}(X) \times \operatorname{Diff}_{G}(Y) \times C_{G}^{\infty}(X, Y) \underset{\Phi}{\longrightarrow} C_{G}^{\infty}(X, Y),
$$

and for each $f \in C_{G}^{\infty}(X, Y)$, we define the corresponding orbit-map

$$
\operatorname{Diff}_{G}(X) \times \operatorname{Diff}_{G}(Y) \underset{\Phi_{f}}{\longrightarrow} C_{G}^{\infty}(X, Y) .
$$

We consider the $G$-bundles $T X, T Y, f^{*} T Y$ and their "invariant sections" $\Gamma^{\infty}(T X)^{G}, \Gamma^{\infty}(T Y)^{G}, \Gamma^{\infty}\left(f^{*} T Y\right)^{G}$. (These are modules over the corresponding rings of $G$-invariant functions.)

As in the usual case [3], [6] we have linear mappings

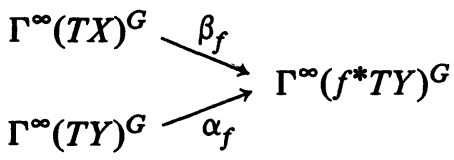

defined in a natural way.

By definition, $f$ is infinitesimally stable if $\alpha_{f}+\beta_{f}$ is surjective.

By definition, $f$ is stable if Image $\Phi_{f}$ is a neighbourhood of $f \in C_{G}^{\infty}(X, Y)$.

With these definitions we have the

StABILITy THEOREM. Let $f \in C_{G}^{\infty}(X, Y)$ be infinitesimally stable. Then:

(i) Whenever $Z_{1}$ is the germ of a metrizable or compact topological space, $Z_{2}$ the germ of a smooth finite dimensional manifold, and $\psi: Z_{1} \times Z_{2} \rightarrow$ $C_{G}^{\infty}(X, Y)$ a $C^{0, \infty}$-germ of a map sending the base points to $f$, there is a germ of a $C^{0, \infty}$ map $\Psi: Z_{1} \times Z_{2} \rightarrow \operatorname{Diff}_{G}(X) \times \operatorname{Diff}_{G}(Y)$ sending the base points to (id $X) \times($ id $Y$ ) and such that

AMS (MOS) subject classifications (1970). Primary $58 \mathrm{C} 25$.

Copyright $\odot$ 1975, American Mathematical Society 


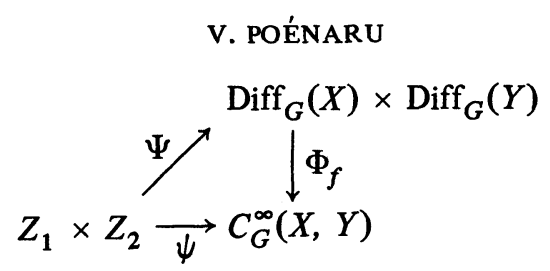

is commutative.

(ii) There is a neighbourhood $f \in N \subset C_{G}^{\infty}(X, Y)$ such that every $f^{\prime} \in N$ is also infinitesimally stable

(iii) $f$ is stable.

The proof relies heavily on the work of J. Mather [2], [3] (which is generalized by this theorem) and of G. Schwarz [7].

2. Let $G \stackrel{\psi}{\longrightarrow} \operatorname{Aut}(V)$ be a linear representation of the compact Lie group $G$.

Let $x \in V$ be the current point of $V$ and $R[x]^{G} \subset R[x]$ be the algebra of $G$-invariant polynomials. According to a classical theorem of Hilbert [1], [8] we can always choose a finite system $\left(\rho_{1}, \ldots, \rho_{k}\right)=\rho \subset R[x]^{G}$ of algebra generators of $R[x]^{G}$. We shall attach to the representation $(G, \psi)$ the number

$$
\operatorname{ord}(G, \psi)=\min _{\rho}\left(\max _{i} \operatorname{deg} \rho_{i}\right) \in Z^{+}
$$

Suppose now $X$ is a (not necessarily compact) $G$-manifold ( $G$ compact). By the slice-representation we have a naturally defined function on the space of orbits $X / G \stackrel{\text { ord }}{\longrightarrow} Z^{+}$.

One of the technical ingredients occurring in the context of the stability theorem is the following

SEMICONTINUITY LEMMA. For every orbit $\{G x\} \in X / G$ there exists a neighbourhood $\{G x\} \in W \subset X / G$, such that, for any $\left\{G x^{\prime}\right\} \in W$, one has

$$
\operatorname{ord}\left(G x^{\prime}\right) \leqslant \operatorname{ord}(G x) \text {. }
$$

This might be useful in the study of deformations of group actions suggested by Thom.

\section{BIBLIOGRAPHY}

1. J. Dieudonné and J. B. Carrel, Invariant theory, old and new, Advances in Math. 4 (1970), 1-80. MR 41 \#186.

2. J. Mather, Stability of $C^{\infty}$ mappings. I. The division theorem, Ann. of Math. (2) 87 (1968), 89-104. MR 38 \#726.

3. - Stability of $C^{\infty}$ mappings. II. Infinitesimal stability implies stability, Ann. of Math. (2) 89 (1969), 254-291. MR 41 \#482.

4. V. Poénaru, Déploiement des fonctions G-invariantes (to appear).

5. - Stabilité structurelle equivariante. I, II (to appear).

6. - Analyse différentielle, Lecture Notes in Math., vol. 371, Springer-Verlag, Berlin and New York, 1974.

7. G. Schwarz, Smooth functions invariant under the action of a compact Lie group, Topology 14 (1975), 63-68.

8. H. Weyl, The classical groups, Princeton Univ. Press, Princeton, N. J., 1939. MR 1, 42. 